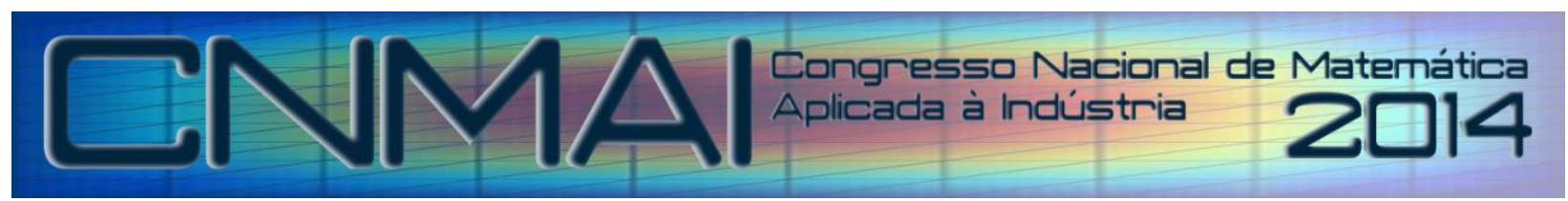

18 a 21 de novembro de 2014, Caldas Novas - Goiás

\title{
DESENVOLVIMENTO DE UM SIMULADOR PARA SISTEMAS COM ACOPLAMENTO ELASTOPLÁSTICO E DIFUSIVO
}

\author{
Felipe Augusto Cruz, felipe84cruz@ hotmail.com $^{1}$ \\ José Maria Andrade Barbosa, jmab13@ gmail.com ${ }^{1}$ \\ Félix Christian Guimarães Santos, flxcgs@ yahoo.com.br ${ }^{1}$
}

${ }^{1}$ Universidade Federal de Pernambuco - Departamento de Engenharia Mecânica, Recife, Pernambuco, Brasil

\begin{abstract}
Resumo: Os simuladores voltados para mecânica computacional são um recurso tecnológico e foram construídos para preverem uma situação da realidade, ou pelo menos tentar se aproximar o máximo possível da mesma. O simulador é uma ferramenta criada que juntamente com simulações, que são configurações variadas para um mesmo simulador, permite avaliar o comportamento de um fenômeno físico que foi anteriormente implementado. O Método do Elemento Finito é uma forma de se obter uma aproximação de um modelo matemático que descreve um comportamento físico. Este método é frequentemente utilizado na área da mecânica computacional, pois é uma importante ferramenta para solução de problemas com equações diferenciais e integrais que são frequentemente utilizadas nos ramos das engenharias. O MPhyScas (Multi-Physics Multi-Scale Solver Environment) é um ambiente computacional dedicado a simular problemas de fenômenos acoplados, ou seja, um conjunto de fenômenos interagindo uns com os outros, levando em consideração o tempo e o espaço. Estes fenômenos podem ser interpretados como sendo as deformações de sólidos, transferência de calor, campos eletromagnéticos, entre outros. Algumas soluções de problemas elásticos, plásticos, transferência de calor, são conhecidas, entretanto quando estes fenômenos interagem mutuamente cria-se um novo problema que geralmente possui uma solução muito mais complexa. Com base neste ambiente, é necessário adquirir programas que possuam a capacidade de trabalhar com vários fenômenos atuantes ao mesmo tempo. $\mathrm{O}$ MPhyScas, por possuir uma estrutura em camadas e fornecer um ambiente dedicado a interação entre vários fenômenos, foi utilizado no desenvolvimento deste trabalho. Este trabalho tem o objetivo de desenvolver simuladores e simulações (utilizando o MPhyScas) para problemas multifísicas. Inicialmente foram criados simuladores e simulações para problemas envolvendo apenas um fenômeno. Após testes realizados e comparações com as soluções analíticas criadas, foram construídos outros simuladores e configurados adequadamente para os problemas envolvendo, simultaneamente, os fenômenos de elasticidade, plasticidade e difusão.
\end{abstract}

Palavras-chave: método do elemento finito, multifísicas, plasticidade, simulação

\section{INTRODUÇÃ̃}

Sempre houve a existência de problemas que atuam mutuamente e com o avanço da engenharia esta divisão de problemas e suas interações têm sido estudada com frequência, alguns destes problemas foram estudados em: (Constantinescu et al. (2004); Zohdi (2004); Woodtli e Kieselbach (2000); Duda et al. (2006) e Santos, Brito e Barbosa (2005)), entre outros. Devido a esta evolução foi capaz de construir simuladores e criar diversas simulações envolvendo estes fenômenos. Juntando-se a isto, esquemas numéricos estão disponibilizados em diversas literaturas, pois para um mesmo problema há diversas combinações existentes.

Os simuladores (Lencastre (2004), e Hoffman e Perondi (2010)) voltados para mecânica computacional são um recurso tecnológico e foram construídos para preverem uma situação da realidade, ou pelo menos tentar se aproximar o máximo possível da mesma. O simulador é uma ferramenta criada que juntamente com simulações, ou seja, configurações variadas para um mesmo simulador, permite avaliar o comportamento de um processo físico que foi anteriormente modelado.

O método do elemento finito (MEF) (Ross (1996), e Santos, Brito e Barbosa (2006)) é uma forma de se obter uma aproximação de um modelo matemático que descreve um comportamento físico. Este método está frequentemente sendo utilizado na área da mecânica computacional, pois é uma importante ferramenta para solução de problemas com equações diferenciais e integrais que são frequentes nos ramos das engenharias. 
O MPhyScas (Multi-Physics Multi-Scale Solver Environment) é um ambiente computacional dedicado a simular problemas de fenomenos acoplados, ou seja, um conjunto de fenomenos interagindo uns com os outros, levando em consideração o tempo e o espaço. Estes fenomenos podem ser interpretados como sendo as deformações em sólidos, Chaboche e Lemaitre (1990), transferência de calor Reddy e Gartling (1994) campos eletromagnéticos, entre outros.

Ele fornece um ambiente que é suficientemente flexível para acomodar todos os níveis computacionais que podem ser encontrados em simuladores baseados no método dos elementos finitos. Eles são estudados e representados em uma arquitetura de camadas e fornece um ambiente dedicado a interação entre vários fenômenos.

O comportamento mecânico dos metais pode ser afetado através do hidrogênio. $\mathrm{O}$ hidrogênio se desloca na rede cristalina gerando enriquecimento da sua concentração localizada em determinados regiões, tornando o material mais sensível à fragilização. Quando um material está sendo fabricado devem ser levados em conta os carregamentos mecânicos (fenômeno elastoplástico) sofridos pelo mesmo, assim, facilitando o movimento do hidrogênio (fenômeno difusivo) no interior da rede cristalina. Estas interações entre fenômenos foram simuladas e testadas utilizando o MPhyScas.

\section{MODELO ELASTOPLÁSTICO E DIFUSIVO}

Esta seção é responsável pela apresentação das equações que modelam o comportamento elastoplástico com difusão utilizados neste trabalho. Para isto é necessário expor cada um dos modelos envolvidos tratando-se de um modelo bidimensional com estado de tensão plana para pequenas deformações.

Inicialmente tem-se o modelo elástico utilizado no desenvolvimento deste trabalho para o caso de um material isotrópico elástico linear juntamente com o critério da tensão de Von Mises, muito utilizada para materiais metálicos, é possível definir e identificar as regiões elástica e plástica nas quais o material está suscetível a permanecer.

A deformação plástica acumulada é responsável por armazenar as informações adquiridas pelo material durante a sua vida e juntamente com análises da função de escoamento, condições de Kuhn-Tucker e consistência estão apresentados nas próximas subseções.

O modelo elastoplástico com difusão envolve endurecimento isotrópico e cinemático. O endurecimento isotrópico é da forma não linear e diz respeito à um aumento da tensão de escoamento do material. Através do endurecimento cinemático verifica-se que a curva de escoamento se translada no espaço das tensões, mantendo o seu formato, tamanho e a orientação da curva inicial.

\subsection{Modelo Elástico}

O comportamento de um material submetido a este modelo pode ser descrito pela lei de Hooke (Saad (2005), onde as tensões $(\boldsymbol{\sigma})$ são determinadas pelas deformações elásticas $\left(\boldsymbol{\varepsilon}^{e}\right)$ através da matriz de rigidez elástica do material $(\boldsymbol{C})$, apresentada abaixo:

$$
\sigma=C \cdot \varepsilon^{e}
$$

Tomando-se como base a equação da lei geral de Hooke expressa na Eq.(1), encontra-se que para o caso de um material isotrópico elástico linear (Saad (2005)), a equação da tensão relacionado com a deformação, apresenta os parâmetros de Lamé $(\mu, \lambda)$ e possui a seguinte relação:

$$
\sigma=2 \mu \varepsilon^{e}+\lambda \operatorname{tr}\left(\varepsilon^{e}\right) \mathbf{I}
$$

Assumindo que a deformação total (ع) do material é composta por parcelas de algumas deformações, faz-se a decomposição aditiva da deformação nas seguintes parcelas: elástica $\left(\boldsymbol{\varepsilon}^{\mathrm{e}}\right)$, plástica $\left(\boldsymbol{\varepsilon}^{\mathrm{p}}\right)$ e de dilatação devido ao processo difusivo $\left(\varepsilon^{\mathfrak{c}}\right)$. Logo, tem-se:

$$
\boldsymbol{\varepsilon}=\boldsymbol{\varepsilon}^{e}+\boldsymbol{\varepsilon}^{p}+\boldsymbol{\varepsilon}^{c}
$$

Por questões de implementação o tensor de tensões está apresentado por $(\boldsymbol{S})$. Reescrevendo o tensor das tensões explicitado na Eq.(2) e substituindo a deformação elástica da Eq.(2) pela definida na Eq.(3), tem-se:

$$
\mathbf{S}=2 \mu\left(\boldsymbol{\varepsilon}-\boldsymbol{\varepsilon}^{p}-\boldsymbol{\varepsilon}^{c}\right)+\lambda \operatorname{tr}\left(\boldsymbol{\varepsilon}-\boldsymbol{\varepsilon}^{p}-\boldsymbol{\varepsilon}^{c}\right) \mathbf{I}
$$

Com o tensor de tensões definido acima em termos dos parâmetros de Lamè e das deformações total $(\boldsymbol{\varepsilon})$, plástica $\left(\boldsymbol{\varepsilon}^{\mathrm{p}}\right)$ e difusiva $\left(\boldsymbol{\varepsilon}^{\mathrm{c}}\right)$ respectivamente, apresenta-se na próxima subseção as simplificações na tensão de Von Mises utilizadas. 


\subsection{Tensão de Von Mises}

O comportamento unidimensional elástico de um material metálico é definido sempre que a tensão aplicada for menor que a tensão de escoamento do material. Neste tipo de comportamento, verifica-se que após ser retirada a tensão aplicada, o material não apresenta deformações, isto é, possui a mesma configuração inicial.

O comportamento unidimensional plástico de um material metálico é definido sempre que a tensão aplicada for igual ou superior a tensão de escoamento. Neste tipo de comportamento, verifica-se que após ser retirada a tensão aplicada, o material apresenta deformações residuais, ou seja, não possui a mesma configuração inicial.

A tensão efetiva $\left(\sigma_{\mathrm{e}}\right)$ em termos das tensões principais para o problema considerado em questão (estado plano de tensão), está apresentado abaixo:

$$
\sigma_{e}^{2}=\sigma_{1}^{2}-\sigma_{1} \sigma_{2}+\sigma_{2}^{2}
$$

A equação apresentada acima é representada no plano de tensões no formato de uma elipse, onde a região no interior da elipse representa a região elástica do material. Sobre a curva há plastificação do material e para esta curva dá-se o nome de superfície de plastificação (Simo e Hughes (2000)). A superfície de plastificação é definida pela função de plastificação $(f)$ que está representada abaixo:

$$
f=\left\|\mathbf{S}_{d e v}\right\|-\sigma_{y}
$$

A função de plastificação é calculada pela diferença entre a norma do tensor desviador de tensões $\left(S_{d e v}\right)$ e da tensão de escoamento do material $\left(\sigma_{y}\right)$. Na próxima subseção encontra-se o conceito de deformação plástica acumulada.

\subsection{Deformação Plástica Acumulada}

A deformação plástica acumulada ( $p$ ) é a responsável por guardar as informações adquiridas pelo material durante a sua vida e é um escalar positivo e pode ser expresso pela integral representada abaixo:

$$
p=\int\left\|\dot{\boldsymbol{\varepsilon}}^{p}\right\| d t
$$

$\mathrm{Na}$ plasticidade associativa, tem-se que a direção de plastificação $(\boldsymbol{N})$, segue a direção normal da superfície de plastificação, no campo de tensões $\sigma$ sendo representada pela seguinte equação:

$$
\mathbf{N}=\frac{\partial f}{\partial \boldsymbol{\sigma}}
$$

A lei da normalidade estabelece que o incremento do tensor da deformação plástica é diretamente proporcional ao gradiente da função de plastificação ou de escoamento relativo ao campo de tensões.

$$
d \boldsymbol{\varepsilon}^{p}=d \alpha \cdot\left(\frac{\partial f}{\partial \boldsymbol{\sigma}}\right)=d \alpha \cdot \mathbf{N}
$$

Na Eq.(9) o termo $d \alpha$, é o multiplicador plástico e indica a intensidade da deformação plástica na direção normal à função de plastificação definido na Eq.(6).

Com a definição do multiplicador plástico, tem-se que as condições de Kuhn-Tucker (Simo e Hughes (2000)) são necessárias, mas não suficientes e estão apresentadas abaixo:

$$
\left\{\begin{array}{c}
f \leq 0 \\
\dot{\alpha} \geq 0 \\
f \dot{\alpha}=0
\end{array}\right.
$$

Para complementar essas condições representadas na Eq.(10), tem-se a condição de consistência que, conjuntamente garantem que um estado de plastificação será mantido, ou seja, o ponto onde houve a plastificação se manterá plastificando no próximo instante. A condição de consistência é conhecida por exigir que o ponto de carregamento permaneça na superfície de escoamento $(f=0)$ durante a deformação plástica e só assim determinar o 
multiplicador plástico $(\alpha)$. Ela pode ser expressa em função da tensão desviadora $\left(\mathbf{S}_{\mathrm{dev}}\right)$, e dos endurecimentos cinemático $(\boldsymbol{X})$ e isotrópico $(Y)$ segundo Khan e Huang (1995).

$$
f\left(\mathbf{S}_{d e v}+d \mathbf{S}_{d e v}, \mathbf{X}+d \mathbf{X}, Y+d Y\right)=\frac{\partial f}{\partial \mathbf{S}_{d e v}}: \mathbf{S}_{d e v}+\frac{\partial f}{\partial \mathbf{X}}: \mathbf{X}+\frac{\partial f}{\partial Y} \cdot Y
$$

Verifica-se então que a condição é escrita para uma mudança incremental da tensão e dos endurecimentos cinemático e isotrópico. Fazendo as análises da condição de Kuhn-Tucker, tem-se:

$$
f \dot{\alpha}=0\left\{\begin{array}{lll}
\dot{\alpha}>0 & e & f=0 \\
\dot{\alpha}=0 & e & f<0
\end{array}\right.
$$

\subsection{Endurecimento Isotrópico e Cinemático}

Para os metais os endurecimentos alteram a curva de plastificação no campo de tensões, como pode ser observado na Eq.(11). Dividindo-se em endurecimento isotrópico e cinemático. O isotrópico diz respeito à um aumento da tensão de escoamento do material, ou seja, a superfície de plastificação $(f=0)$, assim como apresentado na Fig. 1, tem um aumento uniforme em todas as direções resultando em um aumento da sua região de elasticidade (região interna da elipse).

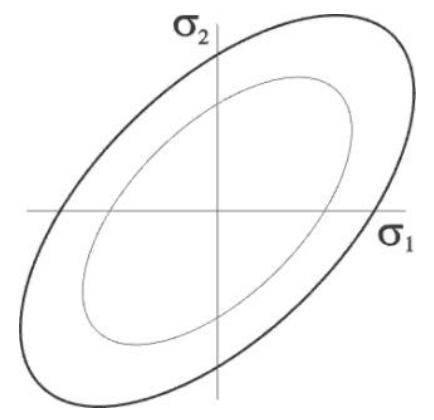

Figura 1. Endurecimento Isotrópico.

Para o problema em questão foi utilizado uma função de endurecimento não linear, em função da deformação plástica acumulada ( $p$ ) Chaboche e Lemaitre (1990):

$$
r(p)=b \cdot\left(1-e^{-d p}\right)
$$

Onde b e d, são parâmetros constitutivos.

Com base nesta equação, a função de endurecimento isotrópico pode ser expressa da seguinte maneira:

$$
Y=r-\sigma_{y}
$$

Para o endurecimento cinemático, a curva de escoamento $(f=0)$ se translada no espaço das tensões, mantém o seu formato, tamanho e a orientação da superfície inicial. Apresenta-se na Fig. 2a um endurecimento cinemático relativo à direção $\sigma_{1}$ e na Fig. $2 b$ é apresentado o endurecimento cinemático relativo à direção $\sigma_{2}$.

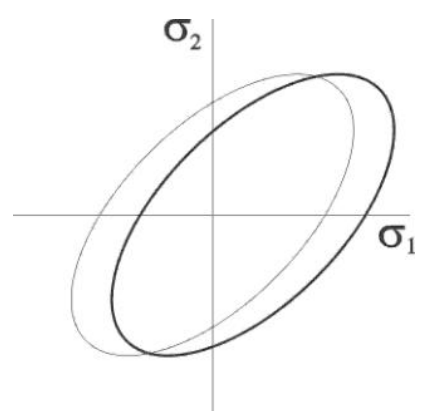

(a) Direção de $\sigma 1$

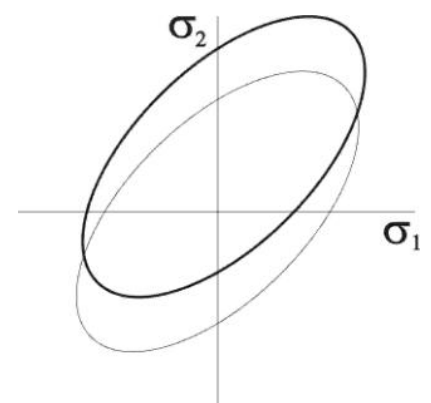

(b) Direção de $\sigma 2$

Figura 2. Endurecimento Cinemático nas direções de $\sigma_{1} \mathrm{e} \sigma_{2}$. 
A equação utilizada para o endurecimento cinemático (Simo, 2000) está apresentada em função de uma função ( $\beta$ ) que possui sua lei de evolução apresentada na Eq.(16).

$$
\begin{gathered}
\mathbf{X}=a \boldsymbol{\beta} \\
\dot{\boldsymbol{\beta}}=\dot{\boldsymbol{\varepsilon}}^{p}-\frac{\phi}{a} \dot{\alpha} \boldsymbol{\beta}
\end{gathered}
$$

Onde $a$ e $\varphi$, são parâmetros constitutivos.

Com a definição dos endurecimentos, a Eq.(6), pode ser reescrita em função da tensão e dos endurecimentos, da seguinte maneira:

$$
f(\mathbf{S}, \mathbf{X}, Y)=\left\|\left(\mathbf{S}_{d e v}-\mathbf{X}\right)\right\|-Y=\sqrt{\frac{3}{2}\left(\mathbf{S}_{d e v}-\mathbf{X}\right):\left(\mathbf{S}_{d e v}-\mathbf{X}\right)}-Y
$$

\subsection{Modelo Difusivo}

Após ter sido apresentado o modelo plástico e conceitos para a deformação plástica, esta subseção está voltada para o modelo difusivo. O modelo químico utilizado neste trabalho, assim como em Brito (2007) altera a matriz de tensões nas componentes hidrostáticas. A deformação química $\left(\varepsilon^{c}\right)$ está apresentada na expressão abaixo:

$$
\boldsymbol{\varepsilon}^{c}=e\left(c-c_{0}\right) \mathbf{I}
$$

Onde $e$, é um parâmetro constitutivo; $c$ e $c_{0}$, são as concentrações de soluto para um instante em determinada posição e concentração de soluto inicial para uma determinada posição, respectivamente.

A equação da taxa de concentração de soluto (Brito (2007)) na matriz sólida é relacionada com o divergente do fluxo $(\boldsymbol{J})$ e está apresentada abaixo:

$$
\begin{gathered}
\dot{c}=-\nabla \cdot \mathbf{J} \\
\mathbf{J}=-D\left(\nabla c-\frac{c}{\alpha} \nabla(\operatorname{tr}(\mathbf{S}))\right)
\end{gathered}
$$

Onde $\dot{c}$, é a taxa de concentração de soluto e $D$ é a difusividade.

Na Eq.(20), verifica-se o acoplamento existente na difusão através da tensão $(\boldsymbol{S})$ dependente da deformação e explicitado na equação abaixo:

$$
\mathbf{S}=\underbrace{\lambda \operatorname{tr}(\boldsymbol{\varepsilon}) \mathbf{I}+2 \mu \boldsymbol{\varepsilon}}_{\sigma_{\text {total }}}-\underbrace{2 e(\lambda+\mu)\left(c-c_{0}\right) \mathbf{I}}_{\sigma_{\text {quimico }}}-\underset{\substack{\sigma_{\text {plasico }} \\ 2 \mu \boldsymbol{\varepsilon}^{p}}}{2 e(\lambda)}
$$

Através da equação acima, identifica-se claramente as parcelas, tanto química, como plástica afetando a tensão, ou seja, um acoplamento fortemente ligado entre fenômenos distintos. Além disto nota-se que através da Eq.(20) o traço da tensão afeta o fluxo relacionado com o fenômeno da difusão afetando o fenômeno da elasticidade e vice-versa através das Eq.(20) e Eq.(19) respectivamente.

\section{IMPLEMENTAÇÃO UTILIZANDO O MPHYSCAS}

Esta seção é responsável por apresentar o algoritmo utilizado para o desenvolvimento de um problema elastoplástico com difusão e posteriormente será feito a analogia entre o algoritmo e as camadas do MPhyScas. O problema em questão possui três fenômenos envolvidos. São eles: Elástico, Plástico e Difusão.

Para a resolução deste problema foi utilizado a técnica chamada de Métodos de Decomposição do Operador (MDO) e Algoritmos de Produtos Associados, que têm demonstrado ser uma alternativa promissora no tratamento de problemas complexos envolvendo equações diferenciais não lineares (Barbosa (1998), e Simo e Mieche (1992)).

O método possui como característica básica decompor aditivamente o operador global em operadores mais simples e então construir algoritmos sequenciais que permitam tratar cada operador em particular de forma independente. Decompõe-se o problema geral em uma sequencia de outros problemas mais simples de forma que possam der 
aplicados métodos numéricos clássicos dos quais se conheça bem o comportamento de estabilidade e convergência. Sendo assim o algoritmo global possui uma aproximação do problema geral e segundo alguns autores (Shaidunov e Marchuk (1983) e, Pinsk, Ortiz e Taylor (1983)), possui propriedades de estabilidade e de consistência se cada algoritmo independente também possuir.

Partindo da decomposição do problema geral, que inicialmente é elastoplástico com difusão, após a decomposição obtém-se 3 problemas que serão resolvidos de forma isolada. Primeiramente será resolvido o problema elástico, em seguida o problema plástico. Considerando que o problema elástico e plástico foram resolvidos, parte-se agora para a solução do problema difusivo.

Lembrando que para a solução do problema plástico os valores de entrada já foram calculados pelo problema elástico, isto também é válido para o problema difusivo, ou seja, possui como situação inicial a solução obtida pelo problema elastoplástico. $\mathrm{O}$ entendimento deste problema pode ser visualizado na Fig. 3 onde observa-se o esquema numérico que trata de uma combinação do problema elastoplástico com difusão.

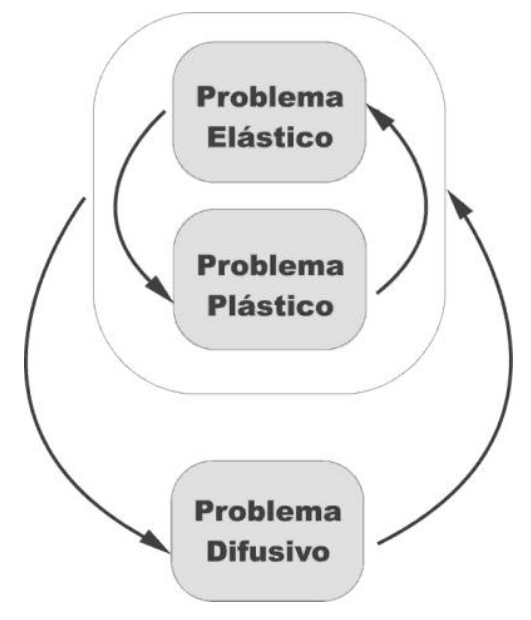

Figura 3. Esquema de solução para o problema envolvendo 3 fenômenos.

Como observa-se na Fig. 3 há três fenômenos envolvendo o problema. Através desta formatação define-se a quantidade necessária de cada objeto nas camadas do MPhyScas, sendo elas tabeladas abaixo:

Tabela 1. Quantidade necessária de cada uma das camadas do MPhyScas (do mais alto nível para o mais baixo nível) para o problema elastoplástico com difusão.

\begin{tabular}{|c|c|}
\hline 01 & Kernel \\
\hline 02 & Block \\
\hline 03 & Group \\
\hline 03 & Phenomenon \\
\hline
\end{tabular}

O Kernel é o responsável pelos laços iterativos globais. Apresenta somente 01 algoritmo e para o problema em questão pode-se ter 02 Blocks, um responsável pela execução dos cálculos e o outro pelo pós-processamento. Esta camada por tratar de laços iterativos globais possui também um parâmetro que representa o intervalo de tempo.

Os Blocks podem possuir mais de 1 algoritmo. O Block responsável pela execução dos cálculos é responsável pelas seguintes funções: inicializações e cálculos do tempo; solução inicial para a difusão; condições iniciais para a elasticidade e difusão; solução até a convergência entre dois instantes consecutivos; solução exata para a elasticidade e difusão; além de cálculos de erros. Já o segundo Block tem a função de fazer a gravação dos dados obtidos.

Assim como definido na Tab. 1 são necessários três Groups, um para a elastoplasticidade, outro para a difusão e outro para a gravação. Para o primeiro grupo (elastoplasticidade) foram definidos alguns conjuntos de tarefas: calcular tamanhos, alocar, inicializar e calcular tempo; estados iniciais e condições de contorno; introduzir condições de contorno, resolver o sistema e calcular o erro da iteração; calcular a plasticidade; solução exata e erro. O Group da difusão possui um grupo de tarefas similar ao da elastoplasticidade. Para finalizar a camada do Group, o último faz a gravação da malha, deslocamento, tensão, plasticidade, difusão, erro e tempo.

Assim como os Groups a camada do Phenomenon também são três. Esta camada é responsável pelo baixo nível e algumas funções são: calcular tamanhos; alocar escalares, vetores e matrizes; inicialização; deletar e realocar matrizes; calcular deslocamentos, tensão, traço, variação no tempo, matriz rigidez, forças plástica e difusiva, matriz massa, concentração de soluto e sua taxa; resolver sistema linear; gravação de malha, deslocamento, concentração de soluto, tempo, plasticidade acumulada; entre outras. 
A representação na estrutura em camadas do MPhyScas está apresentado na Fig. 4. O Kernel $(K)$ é o elemento de mais alto nível, passando pelos Blocks (BO, B1), Groups (GrO, Grl, Gr2) e até chegar aos Phenomenons (PhO, Phl, $P h 2)$ que são os objetos de mais baixo nível.

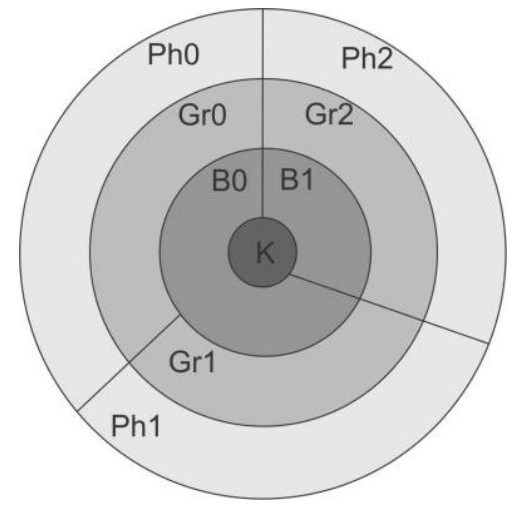

Figura 4. Representação no MPhyScas do problema acoplado elastoplástico com difusão.

Através da Fig.4 constata-se que o Kernel possui dois Blocks; o BO engloba dois Groups e cada um deles seus respectivos Phenomenons. O Block B1, responsável pela gravação, possui somente um Group e seu respectivo Phenomenon.

Na próxima seção encontram-se os resultados e análises obtidos após a criação de um simuladores para sistemas individuais, ou seja, somente um fenômeno (elástico, difusivo, elástico e difusivo) e por fim o problema fim deste trabalho que é o acoplamento elastoplástico com difusão.

\section{RESULTADOS E ANÁLISES}

Para obter os resultados para o problema acoplado do problema elastoplástico com difusão, foram construídos diversos simuladores como os simuladores de elasticidade, difusão e o simulador para o problema elástico difusivo, sendo eles completamente ou parcialmente utilizados para a evolução tanto do MPhyScas, como serviram de referência para outros simuladores mais complexos, como neste caso o simulador para o problema elastoplástico com difusão.

Cada um destes simuladores foram rodados e comparados com resultados analíticos, sendo obtidos resultados satisfatórios. Em cada um dos simuladores criados, manteve-se todos os dados fixos, aumentando somente a quantidade de pontos da malha obteve-se um erro relativo menor e isto era de se esperar, pois os resultados obtidos da solução exata comparados com o método dos elementos finitos, se aproximam a medida que se refina a malha.

\subsection{Problema Elastoplástico com Difusão}

Após a criação de outros simuladores, como: elástico e difusivo, tornou-se mais fácil a construção do simulador em questão, sendo necessário alguns ajustes como criação de novas formas fracas para o acoplamento, além de alterações nos níveis hierarquicamente superiores ao Phenomenon. O problema em análise está apresentado na figura abaixo, em uma placa bidimensional com dimensões de 0,8 por 0,6 unidades de largura e altura respectivamente, com alguns detalhes como um furo de raio 0,1 unidades na coordenada $(0,3 ; 0,3)$ e um detalhe na parte inferior de raio 0,05 unidades na coordenada $(0,3 ; 0,0)$.

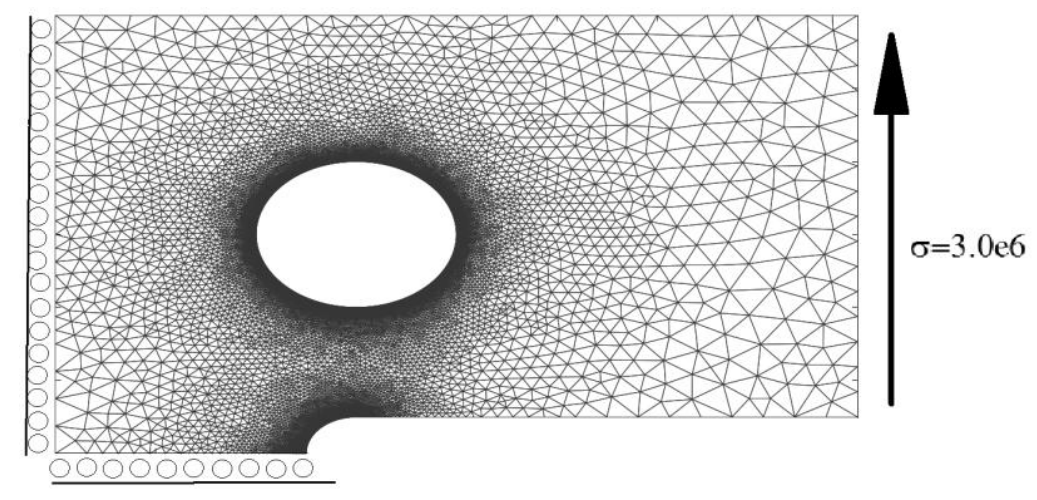

Figura 5. Configuração da placa para o problema elastoplástico com difusão.

A malha apresentada na Fig. 5 contém as seguintes características construtivas: 20.165 vértices; 59.171 arestas e 39.006 faces. Para a sua construção foi selecionado determinados locais para um refinamento, assim como no entorno 
do furo e no detalhe inferior da placa. Para este problema, obtém-se a concentração de soluto sob a placa, tensão de Von Mises, tensão hidrostática e a deformação plástica acumulada para três instantes de tempo para todo o domínio. Os resultados estão apresentados respectivamente nas Fig.6, 7, 8 e 9.

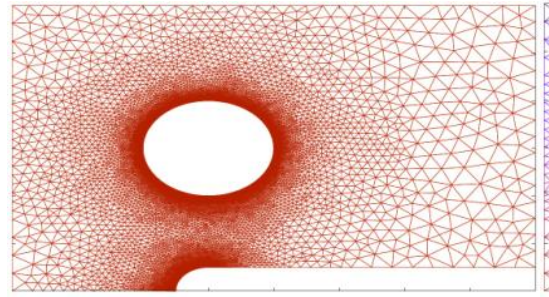

(a) Passo inicial

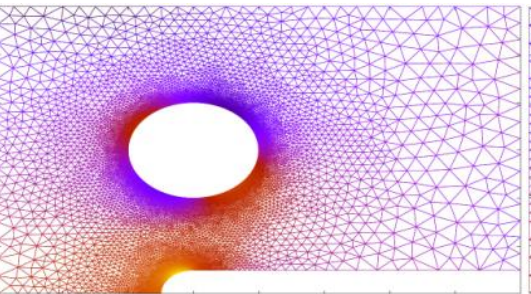

(b) Passo intermediário

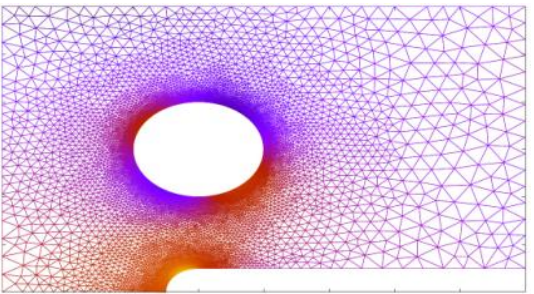

(c) Final

Figura 6. Problema elastoplástico com difusão: Concentração de soluto.

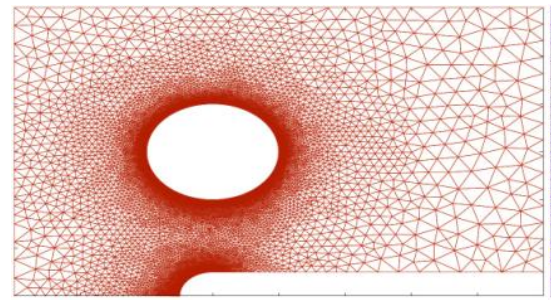

(a) Passo inicial

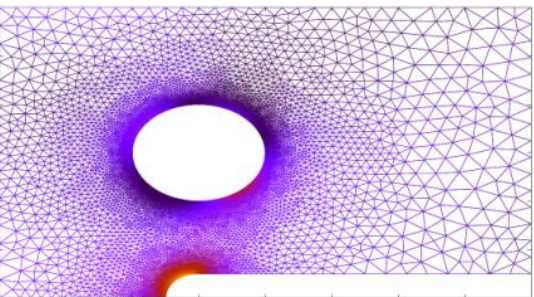

(b) Passo intermediário

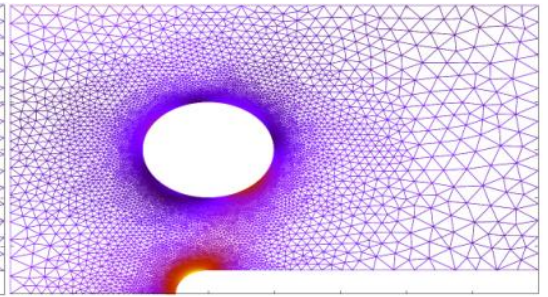

(c) Final

Figura 7. Problema elastoplástico com difusão: Tensão de Von Mises.

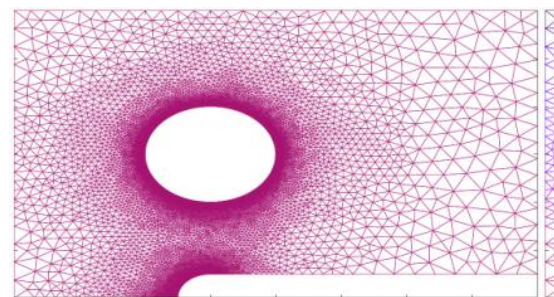

(a) Passo inicial

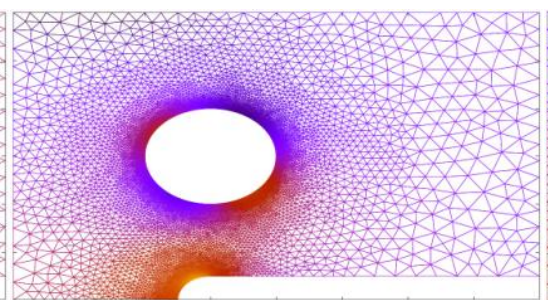

(b) Passo intermediário

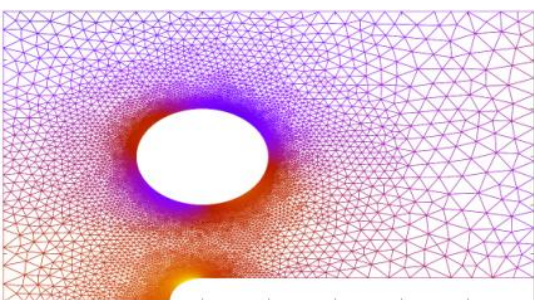

(c) Final

Figura 8. Problema elastoplástico com difusão: Tensão hidrostática.

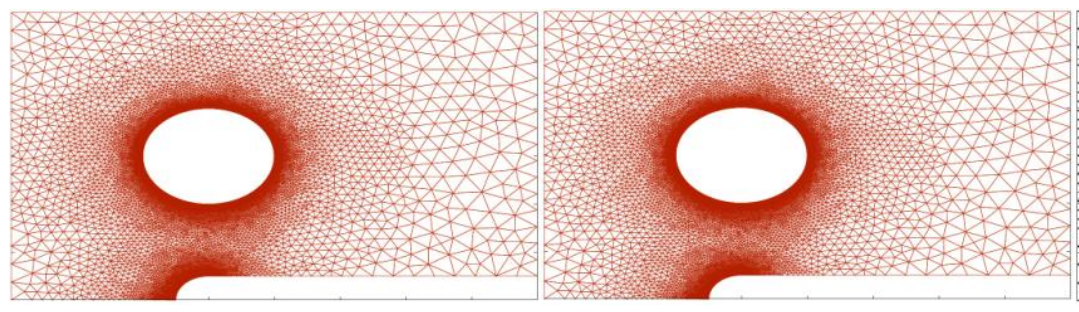

(a) Passo inicial (b) Passo intermediário

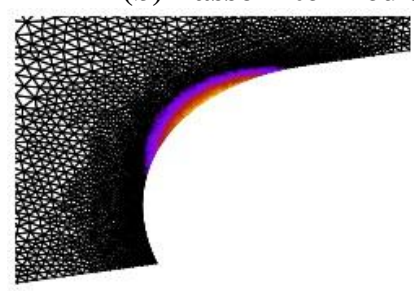

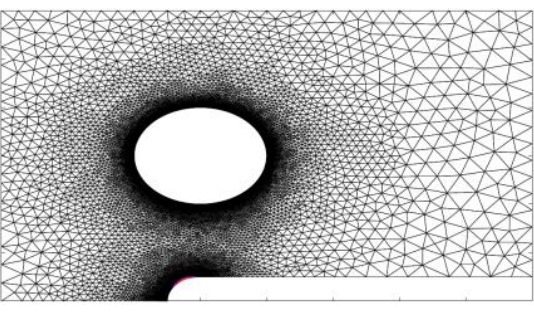

(c) Final

(d) Ampliação da zona plastificada para tempo final

Figura 9. Problema elastoplástico com difusão: Deformação plástica acumulada.

Os valores máximos e mínimos, para o último passo de tempo, da concentração de soluto, Tensão de Von Mises, Tensão Hidrostática e Deformação Plástica Acumulada, encontram-se na Tab. 2.

Tabela 2. Valores Máximos e Mínimos para o problema elastoplástico com difusão.

\begin{tabular}{|l|l|l|}
\hline & Valor Mínimo & Valor Máximo \\
\hline Concentração de Soluto & $2,99 \mathrm{e}-5$ & $3,015 \mathrm{e}-5$ \\
\hline Tensão de Von Mises $(\mathrm{Pa})$ & $0,0 \mathrm{e} 0$ & $1,0 \mathrm{e} 8$ \\
\hline
\end{tabular}




\begin{tabular}{|l|l|l|}
\hline Tensão Hidrostática (Pa) & $-6,0 \mathrm{e} 7$ & $1,0 \mathrm{e} 8$ \\
\hline Deformação Plástica Acumulada & $0,0 \mathrm{e} 0$ & $8,0 \mathrm{e}-5$ \\
\hline
\end{tabular}

Para o problema proposto, notou-se com os resultados obtidos que a concentração do agente químico se deslocou para as posições onde têm-se maiores concentrações de tensão, resultando em uma diminuição de soluto nas regiões onde a tensão é menor. A deformação plástica acumulada encontrada foi muito bem localizada e esperada. Constatou-se que ela vai aumentando com o passar do tempo, o que era de se esperar, pois ela armazena a história do material desde a sua formação.

Na Fig. 9d, tem-se uma ampliação da zona plastificada. A plastificação teve seu inicio em uma região determinada e mantendo as configurações, tem-se o aumento do valor em seus pontos e consequentemente sua região tem um aumento conforme a continuação do carregamento. Foi realizado um teste onde comparou-se a plastificação para o mesmo problema só que não foi considerada a difusão. Para o caso com difusão o maior valor da plastificação foi de 8,0e-5 e desconsiderando a difusão obteve-se 6,0e-5. Conclui-se que o agente químico favorece a plastificação nas regiões onde a tensão é elevada.

Em todas as simulações realizadas e casos analisados tratou-se de utilizar a biblioteca GMM++ (Renard (2010)), pois com ela é possível a criação de vetores e matrizes tanto densos como esparsos, além disto, ela já possui algumas operações e resolvedores de sistemas lineares que foram utilizadas repetidas vezes. No ambiente do MPhyScas são definidos matrizes e vetores totalmente independentes de qual for a biblioteca de entrada, ou seja, pode-se variar de GMM++, para Seldon, SparseLib (Pozo, Remington e Lumsdaine (1996)), IML++ (Dongarra et al. (1995)), entre outras, sem nenhuma outra alteração tornando o sistema fácil de testar e utilizar.

Só é capaz de usar o MPhyScas para problemas acoplados devido a um método implementado que permite fenômenos acessarem campos vetoriais de outros fenômenos, lembrando que este método só é executado após a construção de todos os fenômenos, uma vez que não seria possível acessar campos vetoriais de um campo vetorial de um fenômeno ainda não construído.

\section{AGRADECIMENTOS}

Gostaria de agradecer à Petrobras pelo programa de recursos humanos (PRH-PB 03) pelo fornecimento de uma bolsa de estudo, sem a qual não seria possível finalizar este trabalho.

\section{REFERÊNCIAS}

Barbosa, J.M.A., 1998, "Estudo da Localização da Deformação em Materiais Elasto-Viscoplásticos Levando-se em Conta Efeitos Térmicos e de Inércia", Doutorado em engenharia mecânica, Pontifícia Universidade Católica do Rio de Janeiro, Rio de Janeiro.

Brito, E.R.R.JR., 2007 "Desenvolvimento de um simulador para problemas Multi-físicas", Mestrado em engenharia mecânica, Universidade Federal de Pernambuco, Pernambuco.

Chaboche, J.L. and Lemaitre J., 1990, Mechanics of solids materials.

Constantinescu, A., Charkaluk, E., Lederer G., and L. Verger, 2004, A computational approach to thermo mechanical fatigue, International Journal of Fatigue, (26):805-818.

Dongarra, J., Lumsdaine, A., Pozo, R. and Remington, K., 1995, IML++ v. 1.2, Iterative methods library, Reference guide, university of Notre Dame.

Duda, F.P., Guimarães, L.J., Souza, A.C., and Barbosa, J.M.A, 2006, On the modeling of deformation-diffusiondamage coupling in elastic solids, III European Conference on Computational Mechanics Solids, Structures and Coupled Problems in Engineering.

Hoffman, L.F. and Perondi, L.T., 2010, Estudo de simuladores computacionais aplicados ao ciclo de desenvolvimento de plataformas orbitais.

Lencastre, M., 2004, Conceptualisation of an Environment for the Development of FEM Simulators, Doutorado em ciências da computação, Universidade Federal de Pernambuco, Pernambuco.

Khan, A. S. and Huang, S., 1995, Continuum Theory of Plasticity, Wiley-Interscience Publication.

Pinsk P.M., Ortiz, M. and Taylor, R.L., 1983, Operator split methods for numerical solutions of the elastoplastic dynamic problems, Comp. Meth. Appl. Mech. Engrg., 39:137-157.

Pozo, R., Remington, K.A. and Lumsdaine, A., 1996, SparseLib++ v. 1.5, Sparse matrix class library, Reference guide, University of Notre Dame.

Renard, Y., 2010, Gmm++ user documentation, Release 4.0.

Reddy, J.N. and Gartling, D.K., 1994, The finite element method in heat transfer and fluid dynamics.

Ross, C.T.F., 1996, Finite element programs in structural engineering \& continuum mechanics.

Saad, M.H., 2005, Elasticity - theory, applications and numerics, Elsevier-Academic Press.

Santos, F.C.G., Brito, E.R.R.JR. and Barbosa, J.M.A., 2005, Simulação do problema de evolução do dano em uma barra elasto-viscoplástica com acoplamento termomecânico empregando grafo de interface genérica (gig)., $7^{\circ}$ Congresso Iberoamericano de Engenharia Mecânica.

Santos, F.C.G., Brito, E.R.R.JR. and Barbosa, J.M.A., 2006, Dealing with coupled phenomena in the finite element method. XXVII Latin American Congress on Computational Methods in Engineering, page 461. 
Shaidunov V.V. and Marchuk, G.I., 1983, Difference methods and their extrapolations, Application of Mathematics, 19.

Simo, J.D.C. and Mieche, C., 1992, Associative coupled thermo plasticity at finite strains: Formulation, numerical analysis and implementation, Computer Methods in Applied Mechanics and Engineering, (98):41-104.

Simo, J.C. and Hughes, T.J.R., 2000, Computational inelasticity, Springer.

Woodtli, J. and Kieselbach, R., 2000, Damage due to hydrogen embrittlement and stress corrosion cracking, Engineering Failure Analysis, (7):427-450.

Zohdi, T.I., 2004, Modeling and simulation of a class of coupled thermo-chemo-mechanical processes in multiphase solids, Computer methods in applied mechanics and engineering, (193):679-699.

\title{
DEVELOPMENT OF SIMULATOR FOR SYSTEMS WITH ELASTOPLASTIC COUPLING AND DIFFUSIVE
}

\author{
Felipe Augusto Cruz, felipe84cruz@ hotmail.com ${ }^{1}$ \\ José Maria Andrade Barbosa, jmab13@gmail.com ${ }^{1}$ \\ Félix Christian Guimarães Santos, flxcgs@ yahoo.com.br ${ }^{1}$
}

${ }^{1}$ Federal University of Pernambuco - Department of Mechanical Engineering, Recife, Pernambuco, Brazil

\begin{abstract}
Simulations focused on computational mechanics are technological resource and were built to forecast a real situation, or at least try to bring as much of it. The simulator is a tool created by different configurations that allow the evaluation of one physical process previously modeled. The Finite Element Methods is one way to obtain an approximation of a mathematical model that describes a physical behavior of one particular process. This method is frequently used in computational mechanics methods, because is an important tool for solving problems with differential and integral equations which are very common in engineering areas. MPhyScas (Multi-Physics Multi-Scale Solver Environment) is a computational environment dedicated to simulations of coupled phenomena problems, in other words, a set of phenomena interacting one to another, and considering time and space effects. This phenomena can be interpreted, for example, as deformations in solids, heat transfer and electromagnetic fields. Solutions for elastic, plastic and heat transfer problems, for example, are well know, but a new problem arise when all these phenomena are interacting one to another. The solution for problems with high levels of complexity that involves interactions among multiple phenomena, is not clear and represents one of the major challenges in computational mechanics. Based on this environment, there is a need for software that has the ability to work with several phenomena acting simultaneously. MPhyScas was used to the analysis in this project because it presents layer structure and is dedicated to the set of phenomena interactions. The aim of this dissertation is to develop simulators and simulations (using MPhyScas environment) for multi-physics problems. Simulators and simulations were initially created for problems involving only one phenomenon. After the tests and comparisons with the analytical solutions, other simulators were built and accordingly configured to the problems involving elasticity, plasticity and diffusion phenomena.
\end{abstract}

Keywords: finite element method, multi-physics, plasticity, simulation 